\title{
Response of Josephson-Junction Arrays and Granular Superconductors near the Superconductor-Insulator Transition
}

\author{
Karl-Heinz Wagenblast ${ }^{\mathrm{a}}$, Rosario Fazio ${ }^{\mathrm{a}}$, Anne van Otterlo ${ }^{\mathrm{b}}$, \\ Gerd Schön ${ }^{\mathrm{a}}$, Dario Zappalá ${ }^{\mathrm{c}}$, and Gergely T. Zimanyi ${ }^{\mathrm{d}}$ \\ a Institut für Theoretische Festkörperphysik, Universität Karlsruhe, D-76128 \\ Karlsruhe, Germany \\ b Theoretische Physik, ETH-Hönggerberg, CH-8093 Zürich, Switzerland \\ c Dipartimento di Fisica, Università di Catania, corso Italia 57, 95128 Catania, \\ Italy \\ d Department of Physics, University of California, Davis, CA 95616, USA
}

\begin{abstract}
We study the response of two-dimensional Josephson-junction arrays and granular films of superconducting material near the superconductor-insulator transition. Close to the transition the system is described by a Ginzburg-Landau-Wilson free energy functional for the global superconducting order-parameter. We consider different situations:

First we study the effect of local ohmic shunts. They yield nonohmic dynamics for the order parameter. The conductivity at the transition is nonuniversal within this model.

Then we discuss a boson-fermion model which yields ohmic dynamics for the order parameter. A possible realization for this scenario is the Andreev scattering process at the surface of the superconducting grains. This model leads to a universal conductivity at the transition.

Finally, in the absence of damping we evaluate the universal conductivity in an $\epsilon$-expansion. The result is in good agreement with existing Monte-Carlo data.
\end{abstract}

\section{Introduction}

Granular superconductors and Josephson junction arrays behave similar in many respects. A superconductor to insulator quantum phase transition is observed in both systems [1-4]. It is a direct consequence of the uncertainty relation between phase and number degrees of freedom in a superconductor

Preprint submitted to Elsevier Science 22 November 1995 
[5-10]. In two dimensions the conductance per square, or equivalently the conductivity, is argued to be universal at the transition (i.e. not dependent on microscopic details) $[6,1-4,7-9]$. The experimental evidence is less clear since observed values are sample dependent [3]. This motivated us to reconsider the situation.

In the first part of this article we discuss the influence of dissipation. We use local damping mechanisms which influence the low frequency dispersion of the vortex response in classical arrays [11,12]. Dissipation due to ohmic shunts or quasi-particle tunneling between the islands has been studied in Refs. [13-15]. We describe an array with ohmic shunts to the ground plane, as is realized in proximity-coupled arrays. In this case the Ginzburg-Landau-Wilson (GLW) free energy for the superconducting order-parameter exhibits nonohmic dynamics, which in turn yields a power-law behavior for the conductivity at low frequencies. In this case we find a nonuniversal conductivity at the transition. The influence of a magnetic field is discussed.

Pair breaking processes are another mechanism for damping. These processes are present in inhomogeneous films if the order parameter is locally suppressed, or realized by Andreev scattering at the boundaries of the grains. We use a boson-fermion model [16] to derive an effective GLW description, which in this case exhibits ohmic dynamics.

Finally, in the absence of dissipation we go beyond the Gaussian approximation. We determine the universal conductivity in the $\epsilon$-expansion. The result for the 2-dimensional array is $\sigma^{\star}=0.315\left(4 e^{2}\right) / h$, which is very close to available Monte Carlo data.

\section{Model with Local Ohmic Damping}

The relevant dynamic variables in a Josephson junction array are the phases of the superconducting order parameter on each island. In the presence of ohmic shunts we consider the effective Euclidean action for the array, which reads

$$
\begin{aligned}
S_{\mathrm{eff}}[\varphi]= & \int_{0}^{\beta} d \tau\left[-J \sum_{\langle i j\rangle} \cos \left[\varphi_{i}(\tau)-\varphi_{j}(\tau)-A_{i j}\right]+\frac{1}{8 e^{2}} \sum_{i j} \partial_{\tau} \varphi_{i}(\tau) C_{i j} \partial_{\tau} \varphi_{j}(\tau)\right] \\
& +\frac{1}{2} \int_{0}^{\beta} d \tau d \tau^{\prime} \sum_{i j} \alpha_{i j}\left(\tau-\tau^{\prime}\right)\left[\varphi_{i}(\tau)-\varphi_{j}\left(\tau^{\prime}\right)\right]^{2}
\end{aligned}
$$

The first term describes the Josephson coupling between nearest neighbors. A vector potential is introduced via $A_{i j}=(2 e / \hbar) \int_{i}^{j} \vec{A} d \vec{l}$. The capacitance matrix 
$C_{i j}$ contains diagonal elements $C_{0}$ and off-diagonal elements $C_{1}$, resulting in an interaction of charges which is screened beyond $\sqrt{C_{1} / C_{0}}$, in units of the lattice spacing. For ohmic baths the Fourier transform of $\alpha_{i j}\left(\tau-\tau^{\prime}\right)$ is given by $\left|\omega_{\mu}\right|\left(\alpha_{0}+\alpha_{1} k^{2}\right) / 2 \pi$. We include shunts to the ground $\left(\alpha_{0}=R_{Q} / R_{0}\right)$ and shunts between the islands $\left(\alpha_{1}=R_{Q} / R_{1}\right)$, where $R_{Q}=h /(2 e)^{2}$. The shunts break the $2 \pi$-periodicity in the phase variables since they allow for continuous charge fluctuations.

In the coarse-graining approximation [5] we introduce a complex order-parameter field $\psi$ via a Hubbard-Stratonovich transformation. We decouple the Josephson coupling term and introduce the field $\psi$ such that its expectation value is proportional to that of $\exp (i \varphi)$. This yields the GLW free energy functional

$$
\begin{aligned}
F= & \int d^{2} r d \tau d \tau^{\prime} \bar{\psi}(r, \tau)\left\{\frac{1}{2 J}\left[1+\frac{1}{4}\left(\frac{\vec{\nabla}}{i}+\frac{2 e}{\hbar} \vec{A}\right)^{2}\right]-g\left(\tau-\tau^{\prime}\right)\right\} \psi\left(r, \tau^{\prime}\right) \\
& +\kappa \int d^{2} r d \tau|\psi(r, \tau)|^{4} .
\end{aligned}
$$

Thermodynamic properties are derived from the partition function

$$
Z=Z_{0} \int \mathcal{D}^{2} \psi \exp \{-F[\psi, \bar{\psi}]\}
$$

The dynamics of the field $\psi$ is governed by the phase-phase correlator $g(\tau)=$ $\left\langle\exp \left\{i \varphi_{i}(\tau)-i \varphi_{i}(0)\right\}\right\rangle_{0}$. It is given as an expectation value in a Gaussian action including the capacitive and the dissipative contribution of Eq. (1), which is diagonalized by a Fourier transformation

$$
g(\tau)=\exp \left\{-\frac{1}{\beta N} \sum_{k, \mu \neq 0} \frac{1-\cos \left(\omega_{\mu} \tau\right)}{\omega_{\mu}^{2} C(k) /(2 e)^{2}+\left|\omega_{\mu}\right| \alpha(k) /(2 \pi)}\right\},
$$

with $C(k)=C_{0}+C_{1} k^{2}, \alpha(k)=\alpha_{0}+\alpha_{1} k^{2}$. To evaluate the frequency sum we introduce a cutoff $1 / \tau_{c} \approx e^{2} \alpha_{0} / C_{0}$, with finite $\alpha_{0}$ and $C_{0}$. The correlator decays algebraically in time

$$
g(\tau)=\left|\frac{\beta}{\pi \tau_{c}} \sin \left(\frac{\pi \tau}{\beta}\right)\right|^{-\frac{2}{\alpha}} \quad \text { for } \tau>\tau_{c} ; \quad \alpha=\frac{4 \pi \alpha_{1}}{\log \left(1+4 \pi \alpha_{1} / \alpha_{0}\right)}
$$

The Fourier transform for small frequencies reads

$$
g\left(\omega_{\mu}\right)=g(0)-\eta\left|\omega_{\mu}\right|^{s}-\zeta \omega_{\mu}^{2} \quad \text { with } s=\frac{2}{\alpha}-1 .
$$


Using this expression for $g\left(\omega_{\mu}\right)$, the free energy (2) contains a nonohmic dissipative term $\left(\propto\left|\omega_{\mu}\right|^{s}\right)$ (reducing to ohmic, or 'velocity proportional' damping only in the special case $s=1$ ). This means that an ohmic damping in the quantum phase model yields a nonohmic dynamics for the coarse-grained order-parameter.

We determine the phase boundary in the saddle point approximation with the results shown in Fig. 1 . The critical coupling is given by $J_{\text {cr }}=1 / 2 g(0)$. Increasing damping shifts the phase boundary to smaller values of $J$. At $T=0$ a quantum phase transition is ruled out beyond the critical value $\alpha=2$.

\section{Conductivity}

A directly measurable quantity in these systems is the conductivity, on which we focus in the remainder of this paper. The GLW formulation allows us to determine it in the linear response regime from the functional derivatives of the partition function (3)

$$
\sigma_{a b}\left(\omega_{\mu}\right)=\left.\frac{\hbar}{\omega_{\mu}} \int d^{2} r d \tau \frac{\delta^{2} \ln Z}{\delta A_{a}(r, \tau) \delta A_{b}(0)}\right|_{\vec{A}=0} e^{i \omega_{\mu} \tau}
$$

This allows us to express the conductivity in terms of two and four point Green's functions. In the absence of a magnetic field we have [7]

$$
\begin{aligned}
\sigma\left(\omega_{\mu}\right)=\frac{4 \pi}{R_{Q} \omega_{\mu}}[ & \int \frac{d^{3} q}{(2 \pi)^{3}}\left\langle\psi_{\vec{q}}^{*} \psi_{\vec{q}}\right\rangle \\
& \left.-2 \int \frac{d^{3} q d^{3} p}{(2 \pi)^{6}} q_{x} p_{x}\left\langle\psi_{\vec{q}-\frac{\vec{k}}{2}}^{*} \psi_{\vec{p}+\frac{\vec{k}}{2}}^{*} \psi_{\vec{p}-\frac{\vec{k}}{2}} \psi_{\vec{q}+\frac{\vec{k}}{2}}\right\rangle\right]
\end{aligned}
$$

where the $\vec{q}$ are vectors in the 3 -dimensional space-time and $\vec{k}=\left(0,0, \omega_{\mu}\right)$. In the Gaussian approximation the four point function factorizes, and we obtain for the diagonal part of the conductivity

$$
\sigma\left(\omega_{\mu}\right)=\frac{1}{R_{Q} \omega_{\mu}} \frac{1}{\beta} \sum_{\nu} \int d k k^{3} G\left(k, \omega_{\nu}\right)\left[G\left(k, \omega_{\nu}\right)-G\left(k, \omega_{\nu}+\omega_{\mu}\right)\right],
$$

where $G\left(k, \omega_{\mu}\right)=\left[\epsilon+k^{2}+\zeta \omega_{\mu}^{2}+\eta\left|\omega_{\mu}\right|^{s}\right]^{-1}$. 
The Matsubara sum is conveniently expressed as a contour integral, and the analytic continuation $i \omega_{\mu} \rightarrow \omega+i \delta$ yields the real-frequency dependence

$$
\begin{aligned}
& \sigma(\omega)=\frac{1}{2 \pi R_{Q} \omega} \int_{-\infty}^{\infty} \frac{d x}{1-\mathrm{e}^{-\beta x}} \int_{0}^{\infty} d k k^{3}\left[G^{R}(k, x)-G^{A}(k, x)\right] \times \\
& \times\left[G^{R}(k, x)+G^{A}(k, x)-G^{R}(k, x+\omega)-G^{A}(k, x-\omega)\right] .
\end{aligned}
$$

The advanced and retarded Greens functions are given by

$$
G^{A / R}(k, \omega)=\frac{1}{\epsilon+k^{2}-\zeta \omega^{2}+\eta|\omega|^{s} \cos (\pi s / 2) \pm i \eta|\omega|^{s} \operatorname{sign}(\omega) \sin (\pi s / 2)}
$$

with $\epsilon=1 / 2 J-g\left(\omega_{\mu}=0\right)>0$, and $\mathrm{k}$ has been rescaled.

For low frequencies $\omega \ll \omega_{0}=\sqrt{4 \epsilon / \zeta}$, the real part of the conductivity is given by

$$
\operatorname{Re} \sigma(\omega)=\frac{\eta^{2} \sin ^{2}\left(\frac{\pi}{2} s\right)}{6 \pi \epsilon^{2} R_{Q}} \frac{[,(1+s)]^{2}}{,(2+2 s)}|\omega|^{2 s} .
$$

For arbitrary frequencies the $x$-integral in Eq. (9) can be performed numerically. The results for zero temperature are shown in Fig. 2 and Fig. 3. They show a smeared excitation gap of size $\omega_{0}$ for the real part, while the imaginary part behaves capacitively.

Of particular interest is the d.c. $(\omega \rightarrow 0)$ conductivity at the transition, i.e. for $\omega_{0} \rightarrow 0$ (with $\omega_{0} / \omega \rightarrow 0$ ). The value of the d.c. conductivity at the transition depends on the strength of the ohmic damping as shown in Fig. 4. The inclusion of ohmic shunts to the ground makes the conductivity at the transition a nonuniversal function of $\alpha$ for $\alpha>2 / 3$.

\subsection{Magnetic Field}

Our formulation also allows us to study the influence of a magnetic field. For weak frustration $f \ll 1$ we can use the free energy functional (2) which amounts to neglecting the lattice structure, and therefore also commensurability effects. The magnetic field introduces Landau levels. As a result the transition is shifted, and the response is modified due to the presence of the discrete Landau levels spectrum. The longitudinal conductivity is given by $[10]$ 


$$
\begin{aligned}
\sigma\left(\omega_{\mu}\right)= & \frac{\omega_{c}^{2}}{2 R_{Q} \omega_{\mu} \beta} \sum_{n=0}^{\infty} \sum_{\omega_{\nu}}(n+1)\left[2 G_{\omega_{\nu}, n} G_{\omega_{\nu}, n+1}\right. \\
& \left.-G_{\omega_{\nu}+\omega_{\mu}, n} G_{\omega_{\nu}, n+1}-G_{\omega_{\nu}, n} G_{\omega_{\nu}+\omega_{\mu}, n+1}\right]
\end{aligned}
$$

where $G_{\omega_{\mu}, n}=\left[\epsilon+\omega_{c}(n+1 / 2)+\eta\left|\omega_{\mu}\right|^{s}+\zeta \omega_{\mu}^{2}\right]^{-1}$. In the mean field approximation the phase boundary is given by $\epsilon+\omega_{c} / 2=0$, it is shifted to larger values of the Josephson coupling $J$. The analytic continuation follows the same lines as in the field free case. We explicitly evaluate the real part of the conductivity as shown in Fig. 5. The frequency dependence reflects the underlying Landau-level structure. It is smeared due to the influence of the damping.

\section{Boson-Fermion Model}

In this section we study a model in which the bosonic degrees of freedom are coupled locally to gapless fermions. In arrays on a metallic substrate Andreev tunneling process through tunnel barriers separating the array and the ground plane provides such a mechanism. In inhomogeneous films gapless excitations may be present due to disorder which locally suppresses the gap. We describe each of the grains by the boson-fermion model [16]

$$
H=H_{\mathrm{F}}+H_{\mathrm{B}}+H_{\mathrm{I}}
$$

The fermionic part $H_{\mathrm{F}}$ describes free fermions with a finite density of states at the Fermi surface. The form of the bosonic part $H_{B}$ is of interest later. For the interaction we use a local scattering of bosons $b_{q}$ into pairs of fermions $c_{k}$

$$
H_{\mathrm{I}}=\int \frac{d^{3} k}{(2 \pi)^{3}} \frac{d^{3} q}{(2 \pi)^{3}}\left(\gamma b_{q}^{\dagger} c_{q / 2+k, \uparrow} c_{q / 2-k, \downarrow}+\text { h.c. }\right) \text {. }
$$

If we integrate out the fermions an effective action for the bosons is obtained. Since we assume weak pair breaking, we expand the action up to second order in $\gamma$, which yields

$$
\begin{aligned}
S_{\text {eff }}\left[\phi, \phi^{*}\right]= & \int d \tau\left\{H_{\mathrm{B}}\left[\phi(\tau), \phi^{*}(\tau)\right]-\phi^{*}(\tau)\left(\partial_{\tau}-\mu\right) \phi(\tau)\right\} \\
& -\frac{\gamma^{2} N(0)^{2}}{2} \int d \tau d \tau^{\prime} \alpha\left(\tau-\tau^{\prime}\right)\left(\phi^{*}(\tau) \phi\left(\tau^{\prime}\right)+\text { h.c. }\right) .
\end{aligned}
$$

The partition function is represented as a coherent state path integral, $\phi$ is the complex bosonic field. The nonlocal kernel $\alpha(\tau)=[\pi /(\beta \sin (\pi \tau / \beta))]^{2}$ describes the damping of the boson field. 
These considerations are readily generalized to an array of grains. We include tunneling of bosons between the grains and interactions. The action for the array then reads

$$
\begin{aligned}
S_{\mathrm{eff}}\left[\phi, \phi^{*}\right]= & \int d \tau\left[-t \sum_{\langle i, j\rangle}\left(\phi_{i}^{*}(\tau) \phi_{j}(\tau)+\phi_{j}^{*}(\tau) \phi_{i}(\tau)\right)\right. \\
& \left.+\sum_{i, j} n_{i}(\tau) U_{i j} n_{j}(\tau)-\sum_{i} \phi_{i}^{*}(\tau)\left(\partial_{\tau}-\mu\right) \phi_{i}(\tau)\right] \\
& -\int d \tau d \tau^{\prime} \sum_{i} \frac{\gamma^{2} N(0)^{2}}{2} \alpha\left(\tau-\tau^{\prime}\right)\left(\phi_{i}^{*}(\tau) \phi_{i}\left(\tau^{\prime}\right)+\text { h.c. }\right),
\end{aligned}
$$

where $n=\phi^{*} \phi$.

Insight in the properties of this model near the superconductor-insulator transition is gained by an order-parameter description. A coarse-graining approximation for this model can be performed by decoupling the hopping and the dissipative part by a Hubbard-Stratonovich transformation [17]. This yields the GLW free energy

$$
F[\psi, \bar{\psi}]=\frac{1}{\beta N} \sum_{k, \omega_{\mu}} \bar{\psi}\left\{\epsilon+k^{2}+\eta\left|\omega_{\mu}\right|+\zeta \omega_{\mu}^{2}\right\} \psi+\mathcal{O}\left(|\psi|^{4}\right)
$$

where $\eta=[\gamma N(0)]^{2} / t$. In this model the GLW free energy functional exhibits ohmic dynamics (proportional to $\left|\omega_{\mu}\right|$ ). The conductivity in this model has been discussed in Ref. [10]. The result for the conductivity is contained in those derived in the previous section as the limit $s=1$. At the transition we find the universal value $\sigma^{\star}=\frac{1}{8}\left(\frac{\pi}{2}-\frac{2}{\pi}\right)\left(4 e^{2}\right) / h=0.117\left(4 e^{2}\right) / h$.

Further contact to the model (1) can be made in the limit of a large number of bosons on each grain. Then we can decompose the boson field $b=\sqrt{\rho} \mathrm{e}^{i \varphi}$ and neglect fluctuations in the boson density $\rho$. The effective action (15) translates into that of a JJA with a local damping given by

$$
S_{\text {diss }}[\varphi]=-\rho \gamma^{2} N(0)^{2} \int_{0}^{\beta} d \tau d \tau^{\prime} \sum_{i} \alpha\left(\tau-\tau^{\prime}\right) \cos \left(\varphi_{i}(\tau)-\varphi_{i}\left(\tau^{\prime}\right)\right)
$$

This action is equivalent to the effective action of the Andreev scattering process at a normal-metal to superconductor interface [18]. The tunneling matrix element $T$ in this process is identified by $|T|^{2}=\gamma$. The important difference to the model with ohmic damping (1) is the trigonometric dependence on the phase difference. In the present case the action (17) is $2 \pi$-periodic in the phase, which implies that the charge is quantized in units of $2 e$. 
Another powerful method for evaluating critical quantities is the $\epsilon$-expansion. In this section we briefly present our results for the conductivity in the absence of dissipation. In order to set up the $\epsilon$-expansion we should move away from two-dimensions. We consider a system with $d-1$ spatial dimensions, and Eq. (8) should be rewritten accordingly (the three dimensional vectors should be replaced by d-dimensional ones). The calculation presented here is performed at $\mathcal{O}\left(\epsilon^{2}\right)$ (with $\epsilon=4-d$ ). In dimensions different from two we calculate the conductivity.

It useful to introduce the self-energy $\Sigma(\vec{q})$ and the irreducible four-point vertex , ${ }^{(4)}(\vec{q}, \vec{p}, 0)$ (which is needed to evaluate the four point correlation function in Eq. (8)). The vertex function, ${ }^{(4)}(\vec{q}, \vec{p}, 0)$ and the self energy are related by the Ward identity

$$
\frac{\partial}{\partial q_{i}} \Sigma(\vec{q})=2 \int \frac{d^{d} q}{(2 \pi)^{d}} p_{i} G^{2}(\vec{p}),{ }^{(4)}(\vec{q}, \vec{p}, 0) .
$$

By using this identity, which is related to the underlying gauge symmetry of the model, we can prove that $\rho\left(\omega_{\mu}=0\right)=0$ in the insulating phase. In the superfluid phase this cancelation does not occur due to the presence of the three-point vertex in the theory. In order to get a more suitable expression of $\rho\left(\omega_{\mu}\right)$ for a perturbative analysis, we split the four-point irreducible vertex into five parts, ${ }^{(4)}(\vec{q}, \vec{p}, \vec{k})=,{ }_{0}^{(4)}+,{ }_{s}^{(4)}(\vec{q}+\vec{p})+,{ }_{t}^{(4)}(\vec{q}-\vec{p})+,{ }_{u}^{(4)}(\vec{k})+,{ }_{\text {res }}^{(4)}(\vec{q}, \vec{p}, \vec{k})$ according to the external momenta dependence. Here ${ }_{0}^{(4)}$ has no momentum dependence and, ${ }_{\text {res }}^{(4)}(\vec{q}, \vec{p}, \vec{k})$ is the residual part with momentum dependence different from $\vec{q}+\vec{p}$ or $\vec{q}-\vec{p}$ or $\vec{k}$. The terms, ${ }_{o}^{(4)}$ and, ${ }_{u}^{(4)}(\vec{k})$ do not contribute to the conductivity due to the presence of the factor $q_{x} p_{x}$, while, ${ }_{\text {res }}^{(4)}(\vec{q}, \vec{p}, \vec{k})$ gives a contribution in the $\epsilon$-expansion which is only of order $\mathcal{O}\left(\epsilon^{3}\right)$.

Eq. (18) and the decomposition of the , ${ }^{(4)}$ allow us to express $\sigma\left(\omega_{\mu}\right)$ in the form

$$
\begin{aligned}
\frac{\sigma\left(\omega_{\mu}\right)}{4}= & \frac{2 \pi}{R_{Q} \omega_{\mu}} \int \frac{d^{d} q}{(2 \pi)^{d}}\left[q_{x}^{2}+q_{x} \frac{\partial}{\partial q_{x}} \Sigma(\vec{q})\right] G(\vec{q})[G(\vec{q})-G(\vec{q}+\vec{k})] \\
& -\int \frac{d^{d} q}{(2 \pi)^{d}} \frac{d^{d} p}{(2 \pi)^{d}} q_{x} p_{x} G(\vec{q}) G(\vec{p}) \\
& \left\{[G(\vec{q}+\vec{k})-G(\vec{q})] \gamma^{(4)}(\vec{q}-\vec{p})[G(\vec{p}+\vec{k})-G(\vec{p})]\right. \\
& -G(\vec{p})[2 G(\vec{q}+\vec{k})-G(\vec{q})], \stackrel{(4)}{\text { res }}(\vec{q}, \vec{p}, 0)
\end{aligned}
$$




$$
\left.+G(\vec{p}+\vec{k}) G(\vec{q}+\vec{k}), \underset{\text { res }}{(4)}\left(\vec{q}+\frac{\vec{k}}{2}, \vec{p}+\frac{\vec{k}}{2}, \vec{k}\right)\right\}
$$

where we have introduced $\gamma^{(4)}(\vec{q}-\vec{p})=,{ }_{t}^{(4)}(\vec{q}-\vec{p})-,{ }_{s}^{(4)}(\vec{q}-\vec{p})$. So far no approximations have been used to obtain Eq. (19). The next step is the computation of the self-energy and four-point vertex in the framework of the $\epsilon$-expansion.

At the phase transition the propagator behaves like $G^{-1}(\vec{q})=q^{2-\eta}$ and the value of the critical exponent $\eta$ in $\epsilon$-expansion for the theory considered is known to be $\eta=\frac{\epsilon^{2}}{50}\left(1+\frac{19 \epsilon}{20}\right)+\mathcal{O}\left(\epsilon^{4}\right)$. allowing us to expand the propagator $G(\vec{q})$ in powers of $\epsilon$.

Let us now consider the four-point vertex,${ }^{(4)}(\vec{q}, \vec{p}, \vec{k})$. Using the minimal subtraction renormalization prescription [19], we get

$$
\gamma^{(4)}(\vec{q}-\vec{p})=-\frac{32 \pi^{2} \epsilon}{50}|\vec{q}-\vec{p}|^{\epsilon}
$$

Finally, inserting Eq. (20) into Eq. (19) and discarding terms containing , ${ }_{\text {res }}^{(4)}(\vec{q}, \vec{p}, \vec{k})$ we obtain to order $\epsilon^{2}$

$$
\begin{aligned}
\sigma\left(\omega_{\mu}\right)=\frac{4 e^{2}}{\hbar}\{ & \left(\frac{\epsilon^{2}}{25}-2\right) \frac{,^{2}(d / 2),(1-d / 2)}{\pi^{d / 2} 2^{d},(d)} \\
& \left.-2 z(d) \int \frac{d^{d} y}{y^{4}} f_{\vec{k}}(\vec{y}) f_{\vec{k}}^{*}(\vec{y})\right\} \omega_{\mu}^{1-\epsilon},
\end{aligned}
$$

where

$$
f_{\vec{k}}(\vec{y})=\int_{0}^{1} d \alpha \int_{0}^{\infty} \frac{d \lambda}{(4 \pi)^{d / 2}} \frac{y_{x}}{2 \lambda^{d / 2}} \mathrm{e}^{-\frac{y^{2}}{4 \lambda}}\left[1-\mathrm{e}^{\left(-\alpha(1-\alpha) \lambda-i \alpha y_{z}\right)}\right],
$$

and $z(d)=\left[\pi^{d / 2} /(,(d / 2)) \int_{0}^{\infty} d x x^{d-5}\left(1-(x / 2)^{1-d / 2},(d / 2) J_{(d / 2-1)}(x)\right)\right]^{-1}$. Here,$(x)$ and $J_{\nu}(x)$ are the Gamma and Bessel functions, respectively, and the $\omega_{\mu}$-independent factor in Eq. (21) has been expressed in integral form for the sake of simplicity [20]. The result (21) corresponds to the form predicted by the scaling analysis of Ref. [6]; here it has been computed in an $\epsilon$-expansion.

In dimensions lower than 4 all the integrals are convergent in the ultraviolet regime. In $d \geq 4$ a short-range cutoff should be introduced to regularize the results, but this is not important for our purposes. In two dimension this leads to a value of the universal conductance of

$$
\sigma^{\star}=0.315 \frac{4 e^{2}}{h},
$$


which is close to the results of the Monte Carlo simulations in Ref. [7]. Another analytic calculation of $\sigma^{\star}$ was done using the $1 / N$ expansion [7]. To $\mathcal{O}(1 / N)$ the value of the universal conductance is $0.251\left(4 e^{2}\right) / h$. The $1 / N$ and $\epsilon$-expansion results approach the Monte Carlo value from different sides, but at this stage it is impossible to decide whether they provide an upper ( $\epsilon$-expansion) and lower $(1 / N$-expansion) bound to the exact value.

\section{Conclusions}

We discussed various aspects of the response near the superconductor-insulator transition in Josephson-junction arrays and granular superconductors. In the presence of ohmic shunts to the ground we found an effective order parameter description which exhibits nonohmic dynamics. The conductivity at the transition was found to be nonuniversal in this model. We discussed the influence of a magnetic field. Furthermore we studied a microscopic model which takes into account pair breaking mechanisms. Within this model we find ohmic dynamics of the order parameter and a universal conductivity at the transition, independent of the strength of the pair breaking. In the absence of any dissipative processes we evaluated the universal conductivity in an $\epsilon$-expansion, and find good agreement with Monte Carlo data.

\section{Acknowledgement}

We would like to thank Arno Kampf for valuable discussions. This work is within the research program of SFB 195 and supported by the DFG.

\section{References}

[1] B.G. Orr, H.M. Jaeger, A.M. Goldman, and C.G. Kuper, Phys. Rev. Lett. 56, 378 (1986); H.M. Jaeger, D.B. Haviland, B.G. Orr, and A.M. Goldman, Phys. Rev. B 40, 182 (1989); D.B. Haviland, Y. Liu, and A.M. Goldman, Phys. Rev. Lett. 62, 2180 (1989); Y. Liu, K.A. Greer, B. Nease, D.B. Haviland, G. Martinez, J.W. Haley, and A.M. Goldman, Phys. Rev. Lett. 67, 2068 (1991).

[2] L.J. Geerligs, M. Peters, L.E.M. de Groot, A. Verbruggen, and J.E. Mooij, Phys. Rev. Lett. 63, 326 (1989).

[3] A. Yazdani and A. Kapitulnik, Phys. Rev. Lett. 74, 3037 (1995).

[4] S.-Y. Hsu, J.A. Chervenak, and J.M. Valles, Jr. Phys. Rev. Lett. 75, 132 (1995). 
[5] S. Doniach, Phys. Rev. B 24, 5063 (1981).

[6] M.P.A. Fisher, G. Grinstein, and S.M. Girvin, Phys. Rev. Lett. 64, 587 (1990).

[7] M.-C. Cha, M.P.A. Fisher, S.M. Girvin, M. Wallin, and A.P. Young, Phys. Rev. B 44, 6883 (1991).

[8] E.S. Sørensen, M. Wallin, S.M. Girvin, and A.P. Young, Phys. Rev. Lett. 69, 828 (1992).

[9] M.-C. Cha, and S.M. Girvin, Phys. Rev. B 49, 9794 (1994).

[10] A. van Otterlo, K.-H. Wagenblast, R. Fazio, and G. Schön, Phys. Rev. B 48, 3316 (1993).

[11] H. Beck, Phys. Rev. B 49, 6153 (1994).

[12] S.E. Korshunov, Phys. Rev. B 50, 13616 (1994).

[13] S. Chakravarty, G.-L. Ingold, S. Kivelson, and A. Luther, Phys. Rev. Lett. 56, 2303 (1986); S. Chakravarty, G.-L. Ingold, S. Kivelson, and G. Zimanyi, Phys. Rev. B 37, 3283 (1988).

[14] M.P.A. Fisher, Phys. Rev. B 36, 1917 (1987).

[15] A. Kampf, and G. Schön, Phys. Rev. B 36, 3651 (1987).

[16] J. Ranninger, J.M. Robin, and M. Eschrig, Phys. Rev. Lett. 74, 4027 (1995); R. Friedberg, and T.D. Lee, Phys. Rev. B 40, 6745 (1989).

[17] A. van Otterlo, Ph.D. Thesis, Universität Karlsruhe (1994), (unpublished).

[18] C. Bruder, R. Fazio, and G. Schön, Physica B 203, 240 (1994).

[19] D.J. Amit Field theory, the renormalization group, and critical phenomena, (World Scientific, Singapore) 1984.

[20] R. Fazio and D. Zappalà, preprint. 


\section{FIGURES}

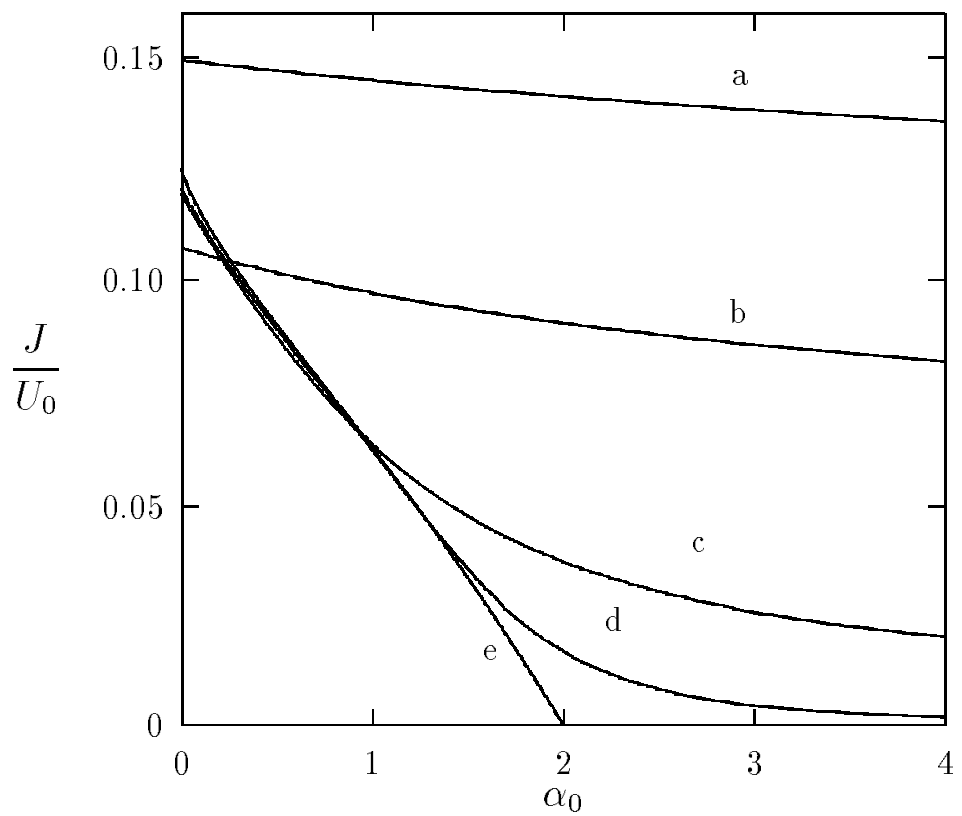

Fig. 1. Phase boundaries as a function of the dissipation $\alpha_{0}$ for different temperatures. a: $T=U_{0} / 5, \mathrm{~b}: T=U_{0} / 10, \mathrm{c}: T=U_{0} / 100, \mathrm{~d}: T=U_{0} / 10000, \mathrm{e}: T=0$, where $U_{0}=4 e^{2} / C_{0}$. With $C_{1}=\alpha_{1}=0$. The superconducting phase lies above the phase boundary.

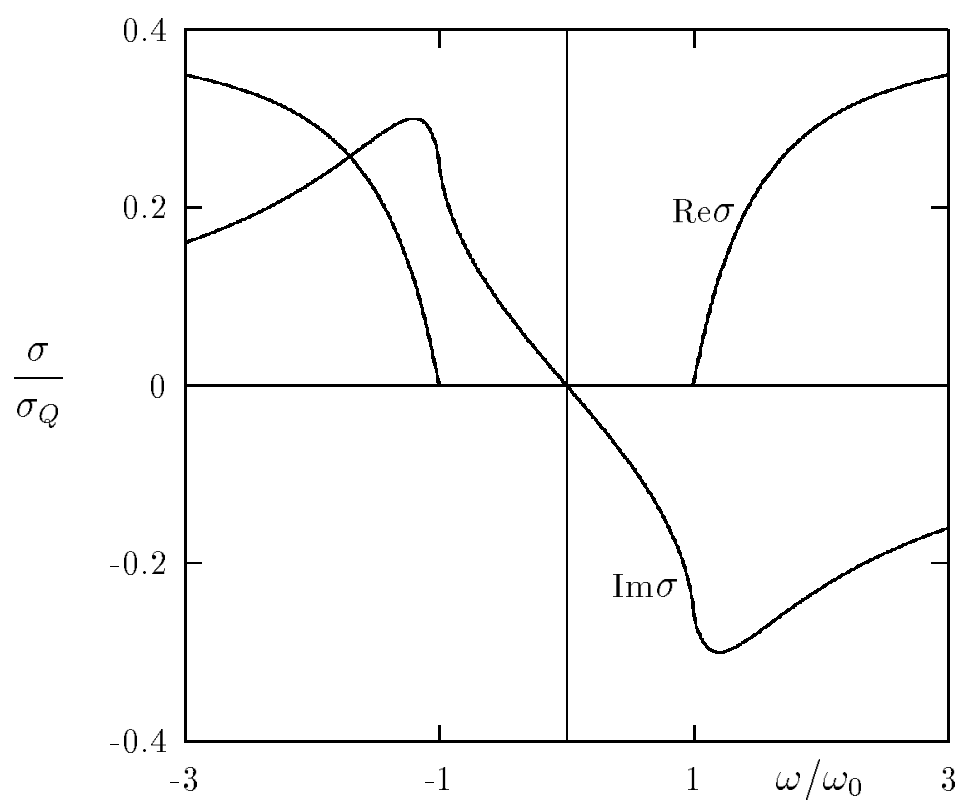

Fig. 2. Real and imaginary part of the conductivity as a function of the frequency without damping, $\sigma_{Q}=4 e^{2} / h$. 


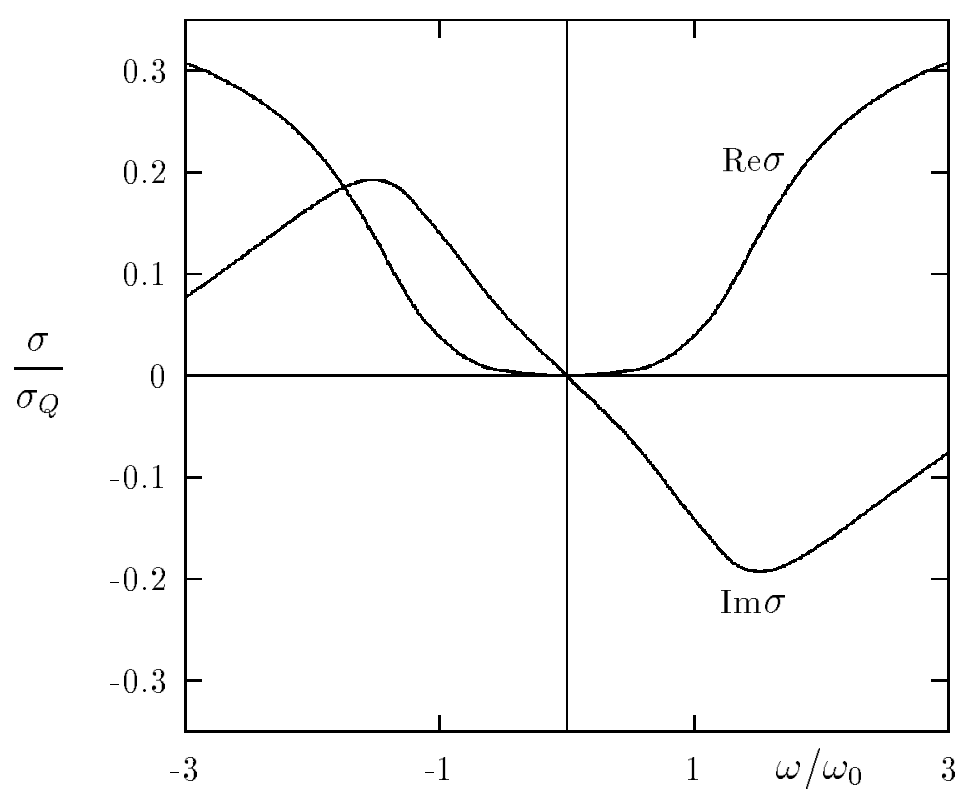

Fig. 3. Real and imaginary part of the conductivity as a function of the frequency with damping, $s=1 / 2, \eta \omega_{0}^{s} / \epsilon=1$.

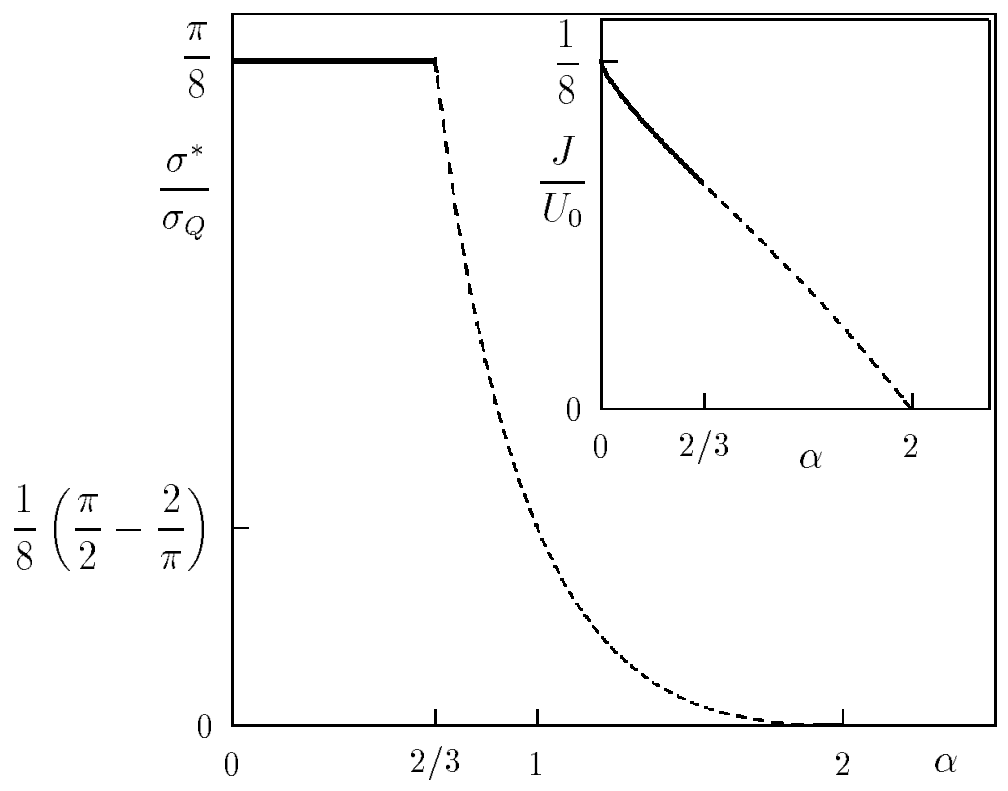

Fig. 4. Conductivity at the transition as a function of $\alpha$ at $T=0$. The inset shows the corresponding phase diagram. Along the solid line the conductivity remains constant, whereas it varies along the dotted line. 


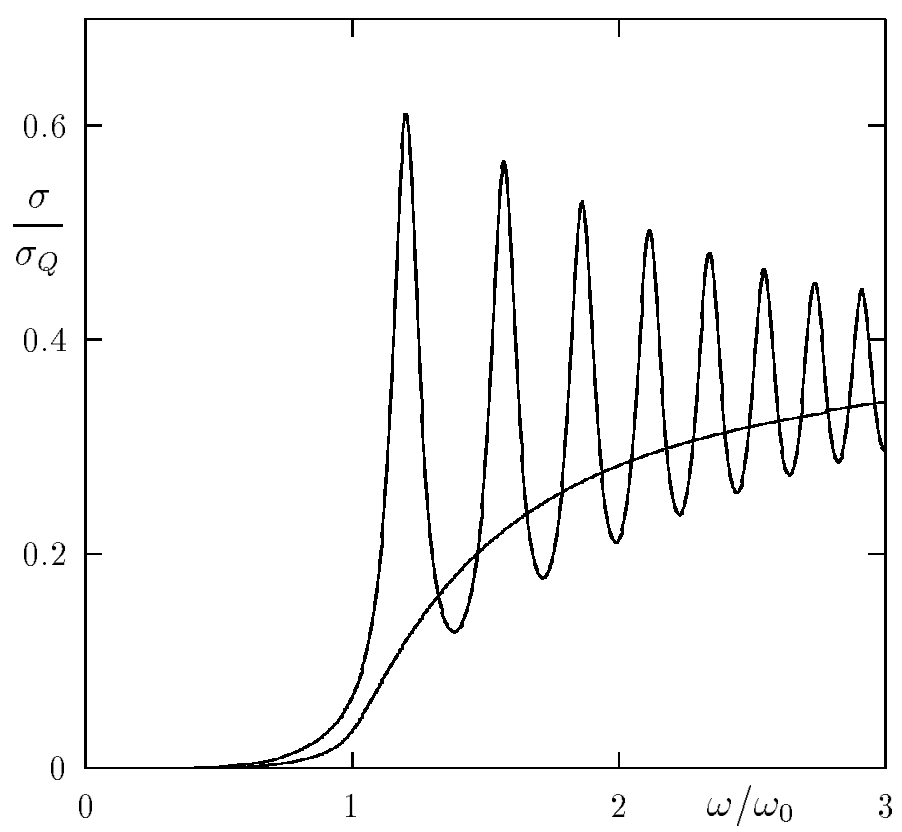

Fig. 5. Real part of the longitudinal conductivity in a magnetic field (oscillating curve), and in zero field (smooth curve). The value of the cyclotron frequency is given by $\omega_{c} / \omega_{0}=1$ and zero, respectively. Damping with $s=1, \eta \omega_{0} / \epsilon=1 / 4$. 